\title{
Evolution of the S-genome in Triticum and Aegilops
}

Badaeva E.D.

Vavilov Institute of General Genetics, RAS, Moscow, Russia

e-mail: kayterinabadaeva@gmail.com

Five diploid Aegilops species of the Sitopsis section: Ae. speltoides, Ae. longissima, Ae. sharonensis, Ae. searsii, and Ae. bicornis, two tetraploid species Ae. peregrina and Ae. kotschyi (Aegilops section) and hexaploid Ae. vavilovii (Vertebrata section) carry different variants of the S-genome. The B- and G-genomes of polyploid wheats are also the derivatives of the S-genome. Evolution of the S-genome species was studied using C-banding and fluorescence in situ hybridization (FISH) with DNA probes representing 5S and 18S-5.8S-26S rRNA genbe families and tandem repeats pSc119.2, pAesp_SAT86, Spelt-1, Spelt-52, pAs1, pTa-535, and pTa-s53. To align the C- and FISH patterns we used the microsatellites $(\mathrm{CTT})_{10}$ and $(\mathrm{GTT})_{9}$, which are major components of the C-heterochromatin in cereals. According to the results obtained, diploid species split into two groups corresponding to Emarginata and Truncata sub-sections, which differ in the C-banding patterns, distribution of rDNAs and other repeats. The B- and G-genomes of polyploid wheat are shown to be most similar to the S-genome of Ae. speltoides. The genomes of allopolyploid wheat evolved as a result of different species-specific chromosome translocations, sequence amplification, elimination and re-patterning of repetitive DNA sequences, which occurred independently in polyploidy wheat and in Ae. speltoides The 5S rDNA locus on chromosome $1 \mathrm{~S}$ was probably lost in ancient Ae. speltoides prior to formation of Timopheevii wheat, but after the emergence of ancient emmer. Evolution of Emarginata species was associated with an increase of C-banding and (CTT) $)_{10}$-positive heterochromatin, amplification of Spelt-52, re-pattering of the pAesp_SAT86, and a gradual elimination of all D-genome-specific repeats. The emergence of Ae. variabilis and Ae. kotschyi did not lead to significant changes of the parental $\mathrm{S}^{*}$-genomes. However, partial elimination of $45 \mathrm{~S}$ rDNA repeats from $5 \mathrm{~S}^{*}$ and $6 \mathrm{~S}^{*}$ chromosomes and alterations of C-banding and FISH-patterns were detected in both tetraploid species. Similarity of the $\mathrm{S}^{\mathrm{v}}$-genome of Ae. vavilovii with the $\mathrm{S}^{\mathrm{S}}$ genome of diploid Ae. searsii confirmed the origin of this hexaploid. A model of the S-genome evolution is suggested. 